\title{
Coevolution in Multispecific Interactions among Free-Living Species
}

\author{
Pedro Jordano
}

Published online: 29 December 2009

(C) Springer Science+Business Media, LLC 2009

\begin{abstract}
Ecological interactions among species are the backbone of biodiversity. Interactions take a tremendous variety of forms in nature and have pervasive consequences for the population dynamics and evolution of species. A persistent challenge in evolutionary biology has been to understand how coevolution has produced complex webs of interacting species, where a large number of species interact through mutual dependences (e.g., mutualisms) or influences (e.g., predator-prey interactions in food webs). Recent work on megadiverse species assemblages in ecological communities has uncovered interesting repeated patterns that emerge in these complex networks of multispecies interactions. They include the presence of a core of supergeneralists, proper patterns of interaction (that resemble nested chinese boxes), and multiple modules that act as the basic blocks of the complex network. The structure of multispecies interactions resembles other complex networks and is central to understanding its evolution and the consequences of species losses for the persistence of the whole network. These patterns suggest both precise ways on how coevolution goes on beyond simple pairwise interactions and scales up to whole communities.
\end{abstract}

Keywords Coevolution · Multispecies interactions . Mutualism · Complex networks · Pollination · Seed dispersal

P. Jordano $(\bowtie)$

Integrative Ecology Group (IEG),

Estación Biológica de Doñana, CSIC,

C/Americo Vespucio s/n, Isla de La Cartuja,

41092 Sevilla, Spain

e-mail: jordano@ebd.csic.es

\section{Introduction}

Complexity is a basic property of the biological world. The fundamental details that surround the natural lives of animals and plants fascinate naturalists but often limit our understanding of the basic principles that drive and generate their diversity. What are the main patterns of interactions among species in megadiverse assemblages, such as the mutualistic interactions among vertebrate frugivores that disperse seeds and plants that produce fleshy fruits in a tropical forest? These interactions can take on a formidable diversity (Fig. 1a), generating complex patterns of mutual dependence among animals and plants that are more than the sum of the pairwise interactions. The complex details of the intimate associations between plants and animals were already recognized by early botanists, like Christian Konrad Sprengel, whose seminal book "Discovery of the secret of Nature in the structure and fertilization of flowers" (Sprengel 1793) presented ample evidence for the importance of crossfertilization in plants. His work was seminal for Darwin's experimental approaches with orchids and the realization of the "entangled bank" of ecological relationships among plants and animals. This has been and continues to be (Thompson 2006) one of the most challenging issues in evolutionary biology: how species coevolve when integrated in complex webs of mutualistic, antagonistic, competitive, or parasitic interactions.

We shouldn't be surprised that empirical naturalists remain skeptical about the new insights that network theory and the formal analysis of complexity bring to the study of biodiversity (Weitz et al. 2007). First, it might be difficult to incorporate research agendas that include a vast amount of multidisciplinary approaches that are far away from our expertise. Second, we need to fully understand how abstract 
a

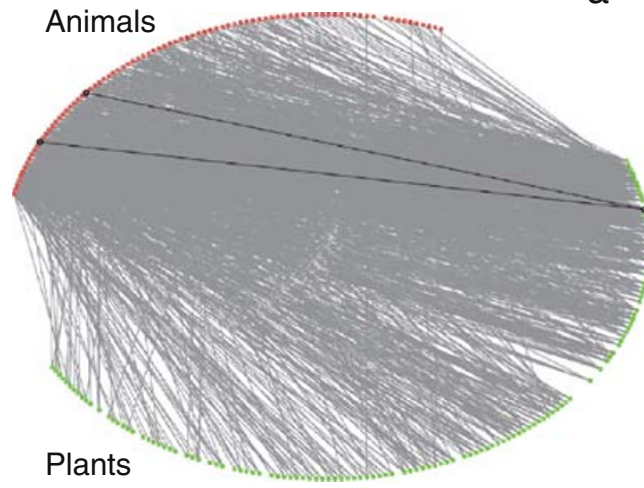

Fig. 1 a A schematic representation of a mutualistic network of interactions among plants producing fleshy fruits and the vertebrate frugivores that disperse their seeds in an Atlantic rainforest locality in SE Brazil (redrawn from Silva et al. 2005). The figure is a bipartite graph, i.e., a representation of the interactions (links) occurring among the species (nodes) in one set (plants) and the species in the other set (frugivorous animals), indicating the interactions that occur in this community. b The basic building blocks of many types of ecological interactions are pairwise relationships of mutual dependence or mutual influence among partner species. Each link in a (highlighted black

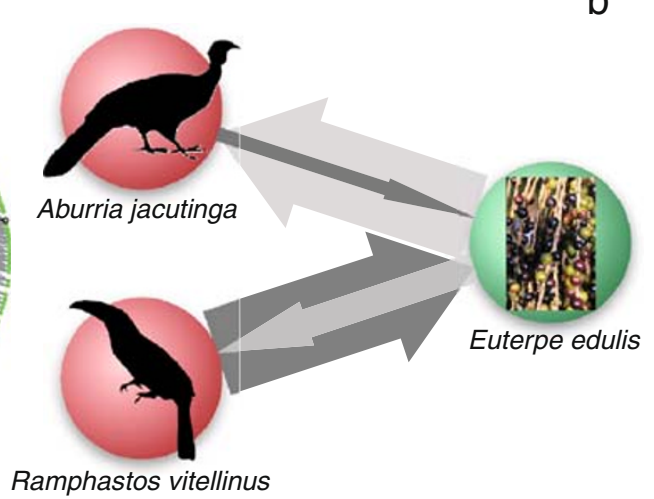

links) actually embeds the relative dependence of a given plant (e.g., palmito Euterpe edulis) on the dispersal service of the frugivore species (e.g., the Jacutinga Aburria jacutinga or the Channel-billed toucan Rhamphastos vitellinus; dark arrow) and the reciprocal dependence of the frugivore on the fruit food resource provided by the plant (light arrow). In this case, the interaction is asymmetrical since the jacutinga depends heavily on palmito fruits while the bird has only a minor contribution to the overall seed dispersal of palmito, which is a keystone resource consumed by a diverse coterie of frugivores in the Atlantic forest (Galetti and Aleixo 1998) representations like network graphs can be embedded with important natural history details of species and their interactions. The analysis of ecological networks is a formal way to visualize, explore, and address the shared patterns that lie beyond the myriad interactions involved in megadiverse multispecies assemblages like tropical forests, coral reefs or soil microorganisms, and plants. We have a formal tool, with a solid multidisciplinary knowledge base, to dissect the complexity of ecological systems by moving from the reductionist analysis of their component parts (e.g., pairwise interactions) to the analysis of their macroscopic properties (Bascompte and Jordano 2007). This essay briefly reviews the principles of network theory applied to ecological systems and considers the new insights gained about the coevolution of megadiverse assemblages of interacting species.

\section{Mutual Benefits, Antagonism, and Who Eats Whom Complexity}

Predator-prey interactions represent an iconic view of ecological webs and have been a central focus of research for years (Pimm et al. 1991). A network view of a food web (Fig. 2a) includes information about the multiple (interactions) links among species (nodes) in the web: who eats whom and the relative magnitude of energy transfers each interaction represents (Dunne et al. 2002). The recent analysis of food webs as complex networks has highlighted basic general principles that influence their stability and the possibilities for recovery after severe disturbances like the suppression of keystone super predators (Jackson et al. 2001) or loss of habitat generalists that compose the core of the interactions (Srinivasan et al. 2007).

The variety of antagonistic, predator-prey, or competitive effects portrayed in food web analysis are among the multiple types of interactions that occur in natural systems (Thompson 1982) and not necessarily the most important ones. Think for example about the keystone relevance of plant-pollinator and plant-frugivore interactions for tropical forests where up to $95 \%$ of the trees and subcanopy shrubs need these animals for effective pollen transfer and successful regeneration (Bawa 1990; Jordano 2000). Forest regeneration would simply collapse without the intervention of animal mutualists. Mutualistic webs of interaction are best depicted as bipartite graphs (Fig. 1a, 2b), where the mutual dependences of each pairwise interaction can be represented. The two distinct sets of species (animals and plants) are linked through coevolved interactions of mutual dependence (Fig. 1b) that depict the reciprocal consequences of their interaction. In the same way as traditional food webs portray the patterns of energy transfer in ecosystems (Fig. 2a), bipartite graphs capture the main elements of coevolved interactions in these multispecific assemblages of species: generalization, asymmetry of mutual dependences, compartments, etc. (Fig. 2b, c). Thus the net outcomes of these interactions, as well as their overall complexity in form (topology) and structure, can be analyzed. Network topology refers mainly to its size (number of nodes) and the form and density of links 

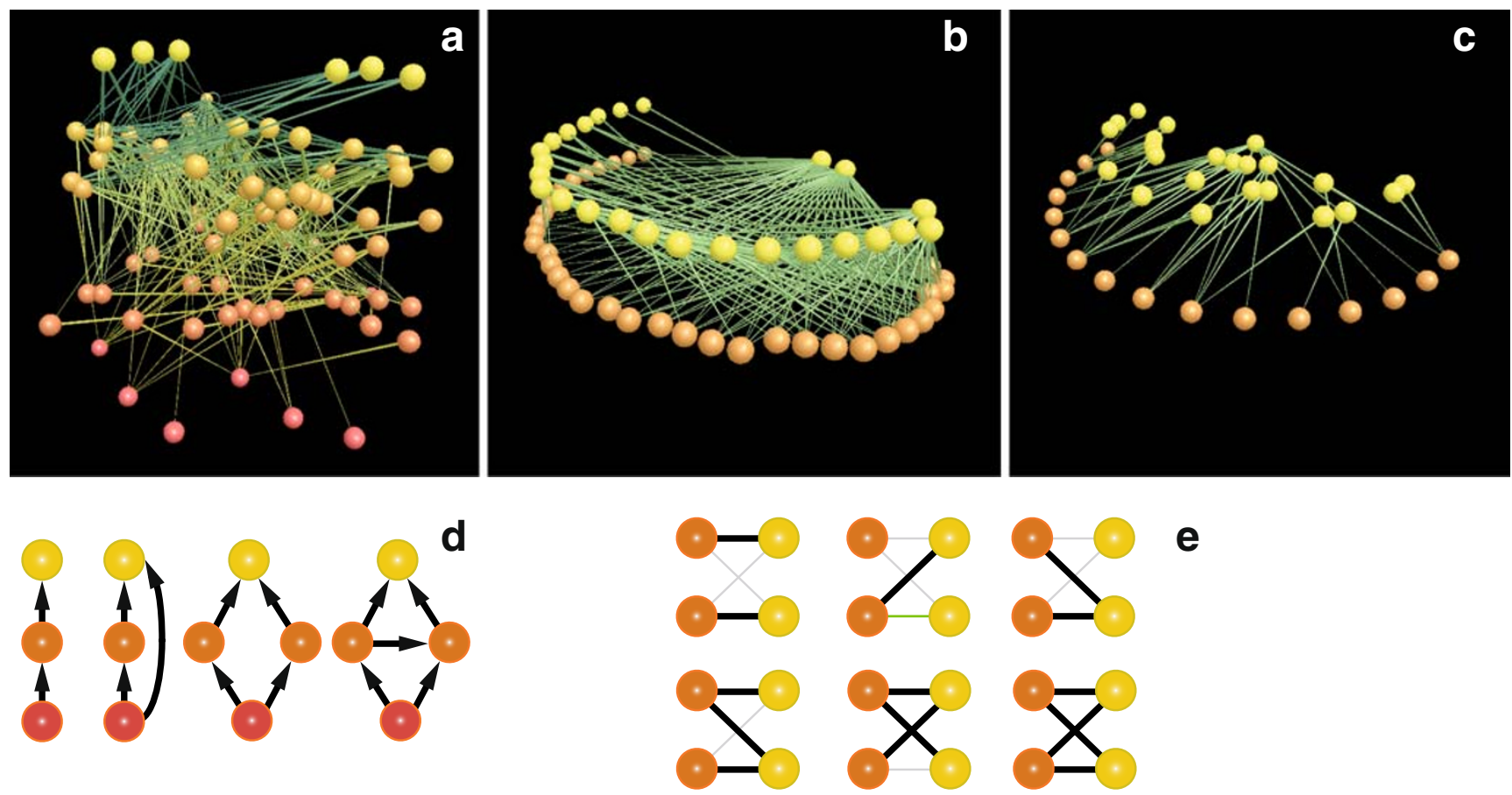

e

Fig. 2 Complex networks of ecological interactions can vary in their shape, link density, and component structure depending on the type of interaction they embed. While food webs typically describe all the interactions occurring in a given ecosystem (a) with multiple trophic levels (Dunne et al. 2002), most plant-animal interactions can be displayed as bipartite graphs (b) describing the pairwise pattern of mutual interdependencies (Jordano 1987) among two distinct sets of animals (orange nodes) and plants (yellow). Interactions among species with a higher degree of intimacy, such as ant-plants show a distinct pattern of structure (c), often with multiple distinct groups (modules) of closely intimate associations (Guimarães et al. 2007).

among them (relative to the maximum possible), as well as their distribution. Two networks might differ in the intensity of mutual effects among species, yet share the same topology. Network structure conveys information about the identity of the nodes: who eats whom and whether distinct subsets of nodes more linked among them exist.

Recent analyses indicate that mutualistic networks have specific signatures in their topology and structure (i.e., the way species are interconnected through mutual dependences) that confer more robustness and stability than expected for randomly assembled interactions (Bascompte and Jordano 2007). Resembling other complex networks (both biological and nonbiotic; Amaral et al. 2000), mutualistic webs are characterized by their heterogeneity (Fig. 2b), with a number of nodes having a high number of interactions and a high number of nodes with few interactions. That is, there are a few super-generalist species that form a well-connected core of the network and many other species with few interactions (Jordano et al. 2003). A randomly assembled web would, in contrast, have a more even distribution of interactions among species, as null
The three types of webs share a complex pattern of interactions made up of multiple simple "building blocks" or motifs (Bascompte and Melián 2005) that vary in shape and frequency across these networks (d, e). Motifs in food webs (d) include simple trophic chains, omnivory, apparent competition, and intraguild predation (from left to right); those in bipartite graphs (e) include different forms of generalization/specialization, with more specialized (e,top) and more generalized (e, bottom) motifs. Images $\mathbf{a}, \mathbf{b}$, and $\mathbf{c}$ produced with FoodWeb3D, written by R. J. Williams and provided by the Pacific Ecoinformatics and Computational Ecology Lab (www.foodwebs.org)

models of mutualistic webs indicate (Vázquez and Aizen 2003). An interesting property of heterogeneous networks is that they are very robust to random disturbances (loss of a node) but very sensitive to selective losses of nodes at the core (Albert et al. 2000). Therefore, mutualistic networks of interaction organized around a distinct subset of super generalists can be reasonably robust to disturbances not directed to this central backbone of their structure.

Three additional signatures of mutualistic networks have been described as characteristic properties, independent of the type of interaction and its geographic setting (Bascompte et al. 2003; Olesen et al. 2007). First, the interactions are nested. If we represent the interaction network (e.g., Fig. 2b) as a matrix, with animal species as rows, and plant species as columns, we can tally the species pairs that interact (cell values of the matrix would be one) or not (cells values as zero). For instance, a series of field censuses similar to used for monitoring the species present in a given area can help us to catalog in matrix form the mutualistic interactions present in a given community (Fig. 3a). If the species in rows and columns are sorted from the most generalist to the most specialist, we can 


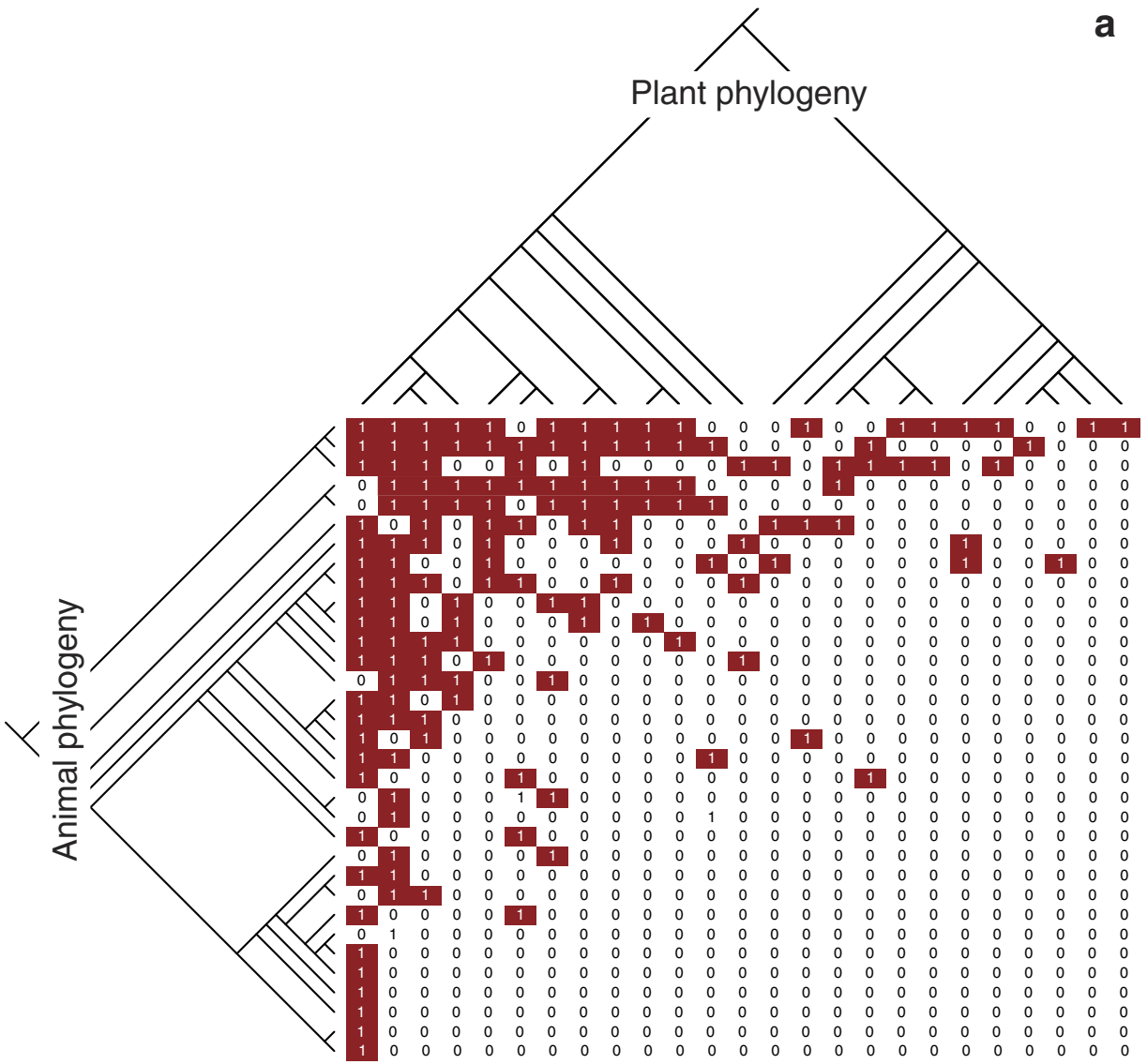

a b
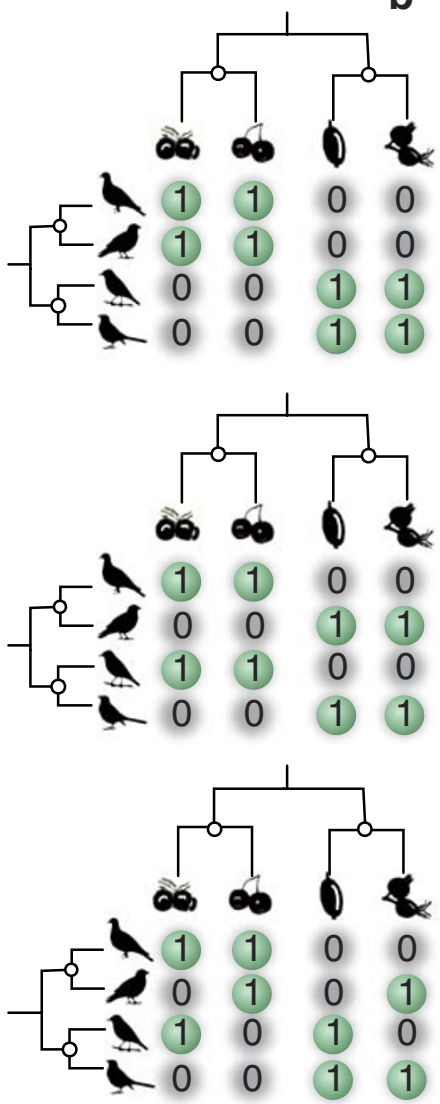

Fig. 3 Phylogenetic patterns are embedded in plant-animal interaction networks and can influence their coevolution. The interaction pattern can be described as a presence-absence matrix denoting the observed and unobserved interactions (as shown here) or a quantitative matrix with data on interaction strength for each observed pairwise interaction. How each species interacts can be affected its evolutionary history (phenotypic traits; a). For example, closely related species might

define the nestedness of the matrix as a deviation from a random pattern and its closeness to a perfectly packed array. That is, how close the matrix resembles perfectly ordered interactions such that each species interactions are subsets of those with which the more generalist species interact (i.e., a situation where all " 1 " in the matrix would be packed to the left of the matrix diagonal, so that " 0 " values remain to the right). The matrix in Fig. $3 \mathrm{a}$ is highly nested. Take the interactions of, say, the third plant species (third column from the left); all of them except one involve animal species with which the more generalized first and second plant species also interact. And if we carry on the comparison for more specialized plants (columns to the right of the matrix), the trend is preserved so that the final pattern (Fig. 3a) is characteristically nested with most interactions mapped in the upper half of the matrix. Bascompte et al. (2003) have shown that plant-pollinator and plant-frugivore assemblages often show nested patterns have a similar pattern of interaction, simply because of niche conservatism (Rezende et al. 2007). Besides, the interaction pattern itself can be subject to the effect of both the animal and plant phylogenetic histories (b), with a marked trend for the interactions to match the phylogenetic history of the two groups (b, top), one of them (the plants in this case; $\mathbf{b}$, middle) or none (b, bottom) Jordano and Bascompte, in prep

(like the one shown in Fig. 3a) with two key properties: the presence of a core of generalists that interact among them and a set of more specialized species that invariably tends to interact with species in the core (i.e., asymmetric specialization). Recent research has shown that nested patterns increase robustness to the loss of species and interactions and favor increased diversity in comparison with randomly assembled mutualistic communities (Bascompte et al. 2003; Burgos et al. 2007; Bastolla et al. 2009).

Second, the wireframe of interactions among species is built on asymmetric and weak reciprocal dependences (Jordano 1987; Bascompte et al. 2006). Just realize that the zero to one records (presence-absence) for the pairwise interactions can take the form of quantitative estimates of the actual strength of dependence of each partner. In Fig. 1b, the strength of mutual dependences between Euterpe edulis and two of its major seed dispersers in the Atlantic rainforest of SE Brazil vary significantly. While the 
palm is quite dependent on the toucan for successful fruit removal, the fruits are a minor part of the toucan generalist diet; in contrast, the jacutinga relies extensively on the palm fruits, but due to low abundance and infrequent visitation to the fruiting palms, it plays a secondary role as seed disperser. This pattern is extremely common in plantanimal mutualisms: most interactions are weak; and the few of them that are stronger tend to be quite asymmetric, with, e.g., the plant depending heavily on a pollinator or frugivore species but the animal being a super-generalist that relies only marginally on that plant. If we sum the total dependences that a plant or an animal species has in a network, we get a measure of the species strength (the quantitative analog to the number of interactions per species in the zero to one matrix). Again, the distribution of strength values is extremely skewed across mutualistic species (Bascompte et al. 2006), with only a few generalists concentrating most of the dependencies for the pollination or seed dispersal services of the rest of the community. Central species thus combine a high number of interactions and a high value of summed dependences (strength) of partner species thus being pivotal for the functioning of the network.

Third, the networks show distinct modules or compartments, i.e., distinct subsets of species that interact more strongly among themselves than with other modules. Olesen et al. (2007) showed that most pollination networks are modular, with distinct subsets of plant-pollinator groups, such as butterfly-pollinated plants or those visited predominantly by hummingbirds. Modules are the basic blocks that structure these networks, analogous to the different walls that made up a building. Individual species can have different roles in this scenario: while some species only interact heavily with species of their own modules, other super-generalists "glue" together all the modules by showing extremely generalized interactions. These interactions tie together peripheral species and can be extremely important for maintaining the cohesiveness of the network. For instance, invasive species can be peripheral in the network during the early stages of invasion but quickly increase in strength and get to the core of the network, displacing native species (Aizen et al. 2008).

All these main properties of the mutualistic networks appear to be omnipresent in nature, independent of the type of interaction or the specific ecosystem or community we study. While enormous progress has been made in recent years to understand the basic patterns and modes of interaction (Bascompte and Jordano 2007), the challenge to understand how these megadiverse assemblages coevolve remains, i.e., how pairwise interactions add up to modules of tightly interacting species to whole communities as diversified as those we can document in tropical forests.

\section{Coevolution of Multispecies Interactions}

What is the basic process assembling these megadiverse networks? Ultimately, the role of each species in the network depends on the number of interactions it establishes with the potential partner species. Established interactions are thus, like the basic blocks that form larger modules that in turn made up the whole complex architecture of the network. These basic blocks are called interaction motifs (Milo et al. 2002), or repeated patterns or forms of interaction, that occur in the network (Fig. 2b, c). Depending on the relative frequency of these different motifs, the overall aspect of the whole network can be very different: more modular and specialized if built predominantly on specialized motifs (e.g., Fig. 2e, top) or more nested and generalized if nonspecialized motifs are dominant (e.g., Fig. 2e, bottom). Data from empirical networks of plant-pollinator and plant-frugivore interactions shows that local abundance has pervasive influence on these patterns, but other important species-specific traits (size, phenology, color) also restrict the range of partners each species interacts with (Jordano 1987; Jordano et al. 2003; Vázquez et al. 2007), determining the type of motifs contributed. A future challenge would be to explore how species-specific traits mold the pattern of interaction and add up to generate these network-wide patterns.

It is far from clear how coevolved selection pressures contribute to the emergence of highly nested patterns of interaction, given the omnipresence of asymmetry of mutual dependencies (and, presumably, asymmetry of phenotypic selection intensities; Jordano 1987; Bascompte et al. 2006). It is expected that the selection pressures originating from pairwise interactions should be more symmetric in antagonistic interactions or in mutualisms with a high degree of intimacy and specialization (like ant-plants; Fig. 2c; Guimarães et al. 2007), resulting in more modular networks with distinct groups of coevolving species. The asymmetric pattern of interaction that pervades mutualistic networks of free-living species favors the diversification and growth of the web by adding new species that link with the core of super-generalists. For instance, rare plant species can probably persist and have a functional service of pollen transfer or seed dispersal by depending strongly on generalist animal partners that, in turn, only marginally rely on the plant resources.

We might expect a variety of influences of the plants' and animals' evolutionary history in shaping network patterns (Fig. 3b). As new species add up in the network, the overall levels of phenotypic convergence and complementarity would increase. For instance, new frugivores would tend to converge (be more similar in morphology) with preexisting frugivore species and share codispersed plants; in turn, selection pressures would increase the 
phenotypic matching of animal traits (body parts, phenology) and fruit traits, increasing complementarity between interacting partners. Convergence would tend to facilitate the persistence of a given species within a multispecific mutualism; complementarity would tend to facilitate how the species efficiently uses the mutualistic services provided by the partners. The interaction pattern will then reflect the phylogenies of the two groups of species (Fig. 3b, top) and markedly deviate from the checkerspot pattern (Fig. 3b, bottom) of interactions expected in the absence of phylogenetic signal. Significant influences of the evolutionary history of only one of the species groups would mean that the group has driven the evolution of the network (Fig. 3b, middle): closely related plants showing a trend to interact with similar sets of frugivore species, but these animal species being not phylogenetically related. A given plant would be using the dispersal services of a wide array of frugivore clades, but a given frugivore species would tend to exploit a subset of phylogenetically restricted fruit species.

When convergence and increased complementarity remain restricted to distinct subsets of species, then modularity will increase through a disproportionate growth of specialized motifs, creating vortexes of coevolutionary change (Thompson 2005, 2006). We can expect these trends for highly intimate mutualistic associations such as ant-plants, symbioses, and high-specificity antagonisms such as host-parasite interactions. In contrast, super generalists are expected to evolve and coevolve within megadiversified webs of interactions among free-living species mainly by evolving abilities to interact with multiple, distinct groups of partners. A characteristic pattern in nested networks of mutualists (Olesen et al. 2007) is that the super-generalists are true hubs in the network, adding interactions that connect different modules. To some extent, the evolution of the supergeneralist lifestyle allows the gluing together of the diverse bricks and blocks that make up the fascinating architecture of these ecological services and their biodiversity.

\section{Concluding Remarks}

The recent development of network-based tools applied to the study of complex patterns of ecological interactions bridges multidisciplinary approaches from statistical mechanics in physics, biocomplexity, ecological modeling, and basic natural history. It is probably the only approach that can successfully decipher the simple, general patterns that lie behind the extreme complexity of interaction webs in ecosystems. A fascinating aspect of these webs is their similarity and analogy to other complex networks, spanning biotic (e.g., gene regulation, cell metabolic reactions) and abiotic (e.g., the internet) scenarios. The multidisciplinary integrative approach to the study of complex networks can be a key to developing early-warning diagnostic criteria to identify critical situations of disturbance in natural areas well before the functional aspects of key ecosystem services, like animal-mediated pollination or seed dispersal, reach a no-return point for their successful restoration. On top of these applied objectives, research on complex ecological networks has taken the first steps to a fuller understanding of how coevolution drives megadiverse assemblages of mutualistic species, which are the backbone of ecosystems like the tropical rainforest.

Understanding complex coevolving networks is important because species and their interactions do not exist in an ecological vacuum. By using new multidisciplinary approaches, we aim to better forecast the risks of losing a single species or collapsing a single interaction to the persistence of the whole ecosystem. We know that these complex systems are more than the sum of their parts, so the consequences of losing one of the parts may extend well beyond its immediate influence. Thus, this understanding will help us to be better prepared to effectively restore the key functions and services needed to rebuild disturbed ecosystems. We need a solid scientific theory of conservation with a knowledge and understanding of complex patterns of biodiversity that at first sight appear impossible to handle and analyze (the "entangled bank"). Coevolving networks of multispecies interactions underpin this entangled bank, and we are just starting to grasp the fine details of their coevolution.

Acknowledgments I appreciate the invitation by John N. Thompson and Rodrigo Medel to contribute to this special issue on coevolution. Their advice and comments, together with one anonymous referee, helped to improve a draft of this paper. Over the years, my ideas on networks have benefited from discussions and collaborations with Jordi Bascompte, Jens M. Olesen, Paulo R. Guimarães Jr., John N. Thompson, Thomas Lewinsohn, and people at the Integrative Ecology Group (Sevilla), especially Alfredo Valido, Carlos Melián, Miguel A. Fortuna, and Jofre Carnicer. My work has been supported by grants from the Spanish Ministry of Science (MICINN; CGL2006-00373) and Junta de Andalucía (P07-RNM2824). This paper is dedicated to Myriam Márquez, for her birthday and the suggestions to design Figs. 1 and 3 .

\section{References}

Aizen M, Morales C, Morales J. Invasive mutualists erode native pollination webs. PLoS Biol. 2008;6:e31.

Albert R, Jeong H, Barabási A. Error and attack tolerance of complex networks. Nature. 2000;406:378-82.

Amaral L, Scala A, Barthelemy M, Stanley H. Classes of small-world networks. Proc Natl Acad Sci USA. 2000;97:11149-52.

Bascompte J, Jordano P. Plant-animal mutualistic networks: the architecture of biodiversity. Ann Rev Ecol Evol Syst. 2007;38: 567-93. 
Bascompte J, Melián C. Simple trophic modules for complex food webs. Ecology. 2005;86:2868-73.

Bascompte J, Jordano P, Melián C, Olesen J. The nested assembly of plant-animal mutualistic networks. Proc Natl Acad Sci USA. 2003;100:9383-7.

Bascompte J, Jordano P, Olesen J. Asymmetric coevolutionary networks facilitate biodiversity maintenance. Science. 2006;312: 431-3.

Bastolla U, Fortuna MA, Pascual-García A, Ferrera A, Luque B, Bascompte J. The architecture of mutualistic networks minimizes competition and increases biodiversity. Nature. 2009;458:101820.

Bawa KS. Plant-pollinator interactions in tropical rain forests. Ann Rev Ecol Evol Syst. 1990;21:399-422.

Burgos E, Ceva H, Perazzo RPJ, Devoto M, Medan D, Zimmermann $\mathrm{M}$, et al. Why nestedness in mutualistic networks? J Theor Biol. 2007;249:307-13.

Dunne J, Williams R, Martinez N. Food-web structure and network theory: the role of connectance and size. Proc Natl Acad Sci USA. 2002;99:12917-22.

Galetti M, Aleixo A. Effects of palm heart harvesting on avian frugivores in the Atlantic rain forest of Brazil. J Appl Ecol. 1998;35:286-93.

Guimarães Jr PR, Rico-Gray V, Oliveira PS, Izzo TJ, dos Reis SF, Thompson JN. Interaction intimacy affects structure and coevolutionary dynamics in mutualistic networks. Curr Biol. 2007;17: 1797-803.

Jackson JBC et al. Historical overfishing and the recent collapse of coastal ecosystems. Science. 2001;293:629-38.

Jordano P. Patterns of mutualistic interactions in pollination and seed dispersal: connectance, dependence asymmetries, and coevolution. Am Nat. 1987;129:657-77.

Jordano P. Fruits and frugivory. In: Fenner M, editor. Seeds: the ecology of regeneration in plant communities. Wallingford, UK: CAB International; 2000. pp. 125-66.
Jordano P, Bascompte J, Olesen JM. Invariant properties in coevolutionary networks of plant-animal interactions. Ecol Lett. 2003;6:69-81.

Milo R, Shen-Orr S, Itzkovitz S, Kashtan N, Chklovskii D, Alon U. Network motifs: simple building blocks of complex networks. Science. 2002;298:824-7.

Olesen J, Bascompte J, Dupont Y, Jordano P. The modularity of pollination networks. Proc Natl Acad Sci USA. 2007;104:19891-6.

Pimm SL, Lawton JH, Cohen JE. Food web patterns and their consequences. Nature. 1991;350:669-74.

Rezende EL, Lavabre JE, Guimarães Jr PR, Jordano P, Bascompte J. Non-random coextinctions in phylogenetically structured mutualistic networks. Nature. 2007;448:925-8.

Silva WR, Guimarães Jr PR, Dos Reis SF, Guimarães P. Investigating fragility in plant-frugivore networks: a case study of the Atlantic forest in Brazil. In: Dennis AJ, Schupp EW, Green RJ, Westcott DA, editors. Seed dispersal: theory and its application in a changing world. Wallingford, UK: CAB International; 2005. p. 561-78.

Sprengel CK. Das entdeckte Geheimniss der Natur im Bau und in der Berfruchtung der Blumen I. Berlin, Germany: Vieweg sen; 1793.

Srinivasan UT, Dunne JA, Harte J, Martinez ND. Response of complex food webs to realistic extinction sequences. Ecology. 2007;88:671-82.

Thompson JN. Interaction and coevolution. New York, US: John Wiley and Sons; 1982.

Thompson JN. The geographic mosaic of coevolution. Chicago, USA: University of Chicago Press; 2005.

Thompson J. Mutualistic webs of species. Science. 2006;312:372-3.

Vázquez DP, Aizen MA. Null model analyses of specialization in plant-pollinator interactions. Ecology. 2003;84:2493-501.

Vázquez DP, Melián CJ, Williams NM, Bluethgen N, Krasnov BR, Poulin R. Species abundance and asymmetric interaction strength in ecological networks. Oikos. 2007;116:1120-7.

Weitz JS, Benfey PN, Wingreen NS. Evolution, interactions, and biological networks. PLoS Biol. 2007;5:e11. 